\title{
Pulsed laser deposition conditions and superconductivity of FeSe thin films
}

\author{
A. Tsukada $\cdot$ K.E. Luna $\cdot$ R.H. Hammond • \\ M.R. Beasley $\cdot$ J.F. Zhao $\cdot$ S.H. Risbud
}

Received: 20 July 2010 / Accepted: 16 November 2010 / Published online: 5 January 2011

(c) The Author(s) 2011. This article is published with open access at Springerlink.com

\begin{abstract}
We report the effects of growth conditions on the superconducting properties of FeSe films epitaxially grown on $\mathrm{LaAlO}_{3}$ substrates by pulsed laser deposition (PLD). Customary materials characterization techniques [X-ray diffraction (XRD), in-situ X-ray photoelectron spectroscopy (XPS), in-situ ultra-violet photoelectron spectroscopy (UPS), and scanning electron microscopy (SEM)] revealed the films had a $c$-axis oriented tetragonal structure with lattice constants dependent on the growth temperature (varied from 100 to $600^{\circ} \mathrm{C}$ ). The standard four-point probe method was used to measure the resistivity and superconducting transitions. Films grown at $400-550^{\circ} \mathrm{C}$ showed a clear superconducting onset but no zero resistance down to
\end{abstract}

\author{
A. Tsukada $(\bowtie) \cdot$ K.E. Luna · R.H. Hammond · M.R. Beasley \\ Geballe Laboratory for Advanced Materials (GLAM), Stanford \\ University, Stanford, CA 94305, USA \\ e-mail: atsukada@rs.kagu.tus.ac.jp \\ K.E. Luna \\ e-mail: kluna@stanford.edu \\ R.H. Hammond \\ e-mail: rhammond@stanford.edu \\ M.R. Beasley \\ e-mail: beasley@stanford.edu \\ A. Tsukada - K.E. Luna - R.H. Hammond - M.R. Beasley \\ Stanford Institute for Materials and Energy Sciences (SIMES), \\ SLAC National Accelerator Laboratory, Menlo Park, CA 94205, \\ USA \\ J.F. Zhao · S.H. Risbud \\ Department of Chemical Engineering and Materials Science, \\ University of California Davis, Davis, CA 95616, USA \\ J.F. Zhao \\ e-mail: jfzhao@ucdavis.edu \\ S.H. Risbud \\ e-mail: srisbud@stanford.edu
}

$2 \mathrm{~K}$. The highest superconducting onset temperature ( $\left.T_{\mathrm{c}}^{\text {onset }}\right)$ of $8 \mathrm{~K}$ was observed in films grown at $500^{\circ} \mathrm{C}$ and the onset temperature was clearly correlated to the ratio of the lattice constants $(c / a)$. As the thickness of the FeSe films increased from $27 \mathrm{~nm}$ to $480 \mathrm{~nm}, T_{\mathrm{c}}^{\text {onset }}$ also increased as the strain in the system was relaxed.

\section{Introduction}

The discovery of superconducting behavior in chemical compounds has a rich history of being intimately connected to materials processing by techniques spanning the gamut from bulk crystal growth and powder sintering to thin film deposition and fiber pulling. The recent introduction of a new class of superconductors based on the iron-pnictide compounds has led to burgeoning efforts directed toward synthesis, characterization and measurement of magnetic and superconducting transitions in these novel high- $T_{\mathrm{c}}$ superconductor systems [1-3]. The report of a superconducting transition at $T_{\mathrm{c}}=26 \mathrm{~K}$ in LaOFeAs first triggered the search for new compounds based on the structure of ironpnictides with the potential for exhibiting superconducting behavior. With the successful synthesis and processing of newer compounds in this family of materials, $T_{\mathrm{c}}$ values above $50 \mathrm{~K}$ [4-6] have been reported in parallel with characterization of different crystal structures in several of these compounds, e.g., $\mathrm{BaFe}_{2} \mathrm{As}_{2}$ [7], $\mathrm{LiFeAs}$ [8], and FeSe [9].

Pulsed laser deposition (PLD) is perhaps the most studied and extensively used materials processing technique for making thin films of complex superconductors. Yet, the synthesis results are often mixed and seem highly dependent on specific chemistry and composition. For example, in the LaOFeAs system, epitaxial thin films were obtained but superconducting properties were poor [10-12]. On the other 
hand, in the case of the $\mathrm{BaFe}_{2} \mathrm{As}_{2}$ system, it was reportedly easy to grow and cleave single crystal samples as well as to deposit high quality thin-films [12, 13]. Furthermore, for the LiFeAs system, successful thin-film growth has yet to be realized. The FeSe system has the simplest crystal structure, consisting of only one FeSe layered block, thus making it an appealing material to study the effect of processing parameters on film growth and structure. Previous reports of thin film growth of FeSe and FeTe materials include a study of thickness dependence on superconducting properties of $\mathrm{FeSe}_{1-x} \mathrm{Te}_{x}$ films [14], substrate dependence on superconducting properties of FeSe films, and suppression of superconductivity by tensile strain in films. In the present work, we report results of PLD of thin films grown from a dense FeSe target (sintered by spark plasma sintering) and the effects of the growth parameters on the lattice constants of the tetragonal structure, stoichiometry, strain in the films, and the superconducting properties.

\section{Experiments}

The FeSe thin films for our temperature dependence study were grown in a UHV chamber on (100) $\mathrm{LaAlO}_{3}$ (LAO) substrates, which is the substrate of choice reported by Nie, using pulsed laser deposition (PLD). For PLD, a Lambda Physik LPX $210 \mathrm{KrF}$ excimer laser was used that produces a 248-nm-wavelength beam with a typical pulse length of 20-30 ns. The energy density on the target was kept around $1.3 \mathrm{~J} / \mathrm{cm}^{2}$ with a repetition rate of $10 \mathrm{~Hz}$ and growth rate of $0.11 \AA$ As. The typical thickness of the films grown in vacuum $\left(\sim 10^{-8}\right.$ torr) was $40 \mathrm{~nm}$. The growth temperature $\left(T_{\mathrm{s}}\right)$ was varied from 100 to $600^{\circ} \mathrm{C}$.

In an effort to speed up the turnover rate of production, we subsequently studied the thickness dependence of these films on LAO in a different HV chamber $\left(\sim 10^{-7}\right.$ torr) using the same excimer laser, with an energy density around $1.67-2.0 \mathrm{~J} / \mathrm{cm}^{2}$, a repetition rate of $1 \mathrm{~Hz}$, a growth rate of $0.08 \AA / \mathrm{s}$, a target to substrate distance of $41 \mathrm{~mm}$, and a growth temperature of $400^{\circ} \mathrm{C}$. We used the same target as used in the UHV chamber to make films of thickness 27,80 , and $165 \mathrm{~nm}$ and also a new target, made of the same initial powder, to make films of thickness $127,194,372,480 \mathrm{~nm}$. The thickness measurement of the $27 \mathrm{~nm}$ film was measured by reflectivity and the remaining films were measured using a profilometer.

Our target used commercially available FeSe powder (99.9\%) pressed into a disk by the spark plasma sintering technique [15]. The films were characterized by $\mathrm{X}$-ray diffraction (XRD), X-ray photoelectron spectroscopy (XPS), ultra-violet photoelectron spectroscopy (UPS), scanning electron microscope (SEM), and resistivity measurements. The XPS and UPS measurements were performed in

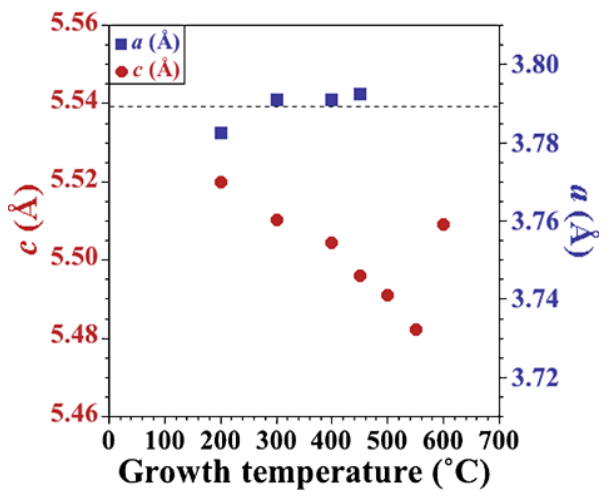

Fig. 1 The $c$-and $a$-axis values of FeSe films as a function of growth temperature. The broken line is the $a$-axis value of the LAO substrate

situ. The photon source for XPS is $\operatorname{MgK}_{\alpha}$ and for UPS the $\mathrm{HeI}(21.2 \mathrm{eV})$ line was used.

\section{Results and discussions}

With the ability to create $c$-axis oriented films with good surface morphology, as we show later in this paper, we focused our attention on characterizing the effects of the temperature dependence during deposition on physical quantities of interest. We do not determine the stoichiometry of the thin films explicitly because the stoichiometry of the PLD target does not always correspond to the stoichiometry of the film. In addition, determining the stoichiometry directly from a film to such extreme accuracy is difficult when such tiny variations exists, as shown by McQueen et al. that $\mathrm{Fe}_{1.01} \mathrm{Se}$ is superconducting while $\mathrm{Fe}_{1.03} \mathrm{Se}$ is not. However, we do follow a remarkable trend discovered by McQueen et al., namely that the ratio of the lattice constants $c / a$ in $\mathrm{Fe}_{1.01} \mathrm{Se}$ linearly varies with $T_{\mathrm{c}}$ [16]. Thus this quantity is a good parameter to track to achieve a higher $T_{\mathrm{c}}$.

Figure 1 shows the variation of $a$ - and $c$-axis lattice constants of the FeSe films as a function of growth temperature. Due to strain from the lattice mismatch, the $a$-axis values deviate from the bulk values of $\mathrm{FeSe}_{1-x}(3.767 \sim 3.779 \AA)$ and coincide with the $a$-axis value of the LAO substrates (3.788 $\AA$ ). The $c$-axis value of FeSe films strongly depends on growth temperature, and as can be seen in the figure, monotonically decreases with increasing growth temperature from $5.52 \AA$ for films grown at $T_{\mathrm{S}}=200^{\circ} \mathrm{C}$ to $5.48 \AA$ for films grown at $T_{\mathrm{S}}=550^{\circ} \mathrm{C}$. While the reported $c$-axis values for bulk FeSe are still in question, our highest value of $5.52 \AA$ coincides with the value reported by some groups [16-18], and our lowest value of $5.48 \AA$ coincides with the value reported by Yeh [9]. With regards to the structure, Nie reported that hexagonal $\mathrm{FeSe}$ tends to grow at a high growth temperature $\left(T_{\mathrm{S}}=450-800^{\circ} \mathrm{C}\right)$ [19]. In our films, however, the XRD patterns show no trace of hexagonal FeSe, even 
Fig. 2 Resistivity as a function of temperature for FeSe films grown at different temperatures

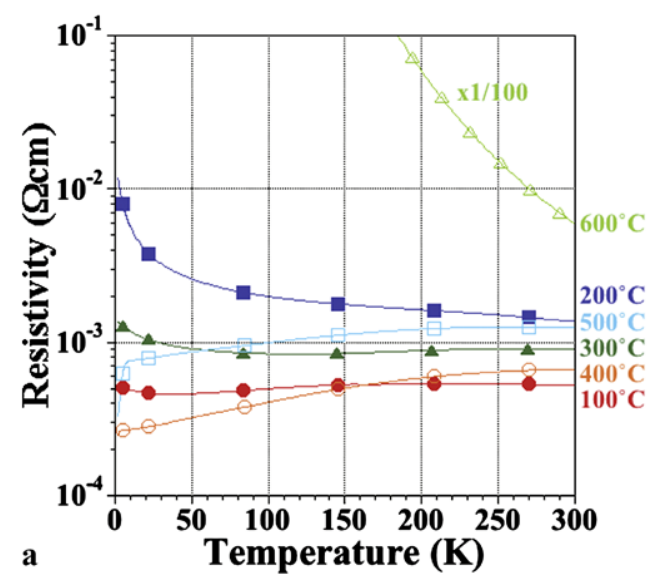

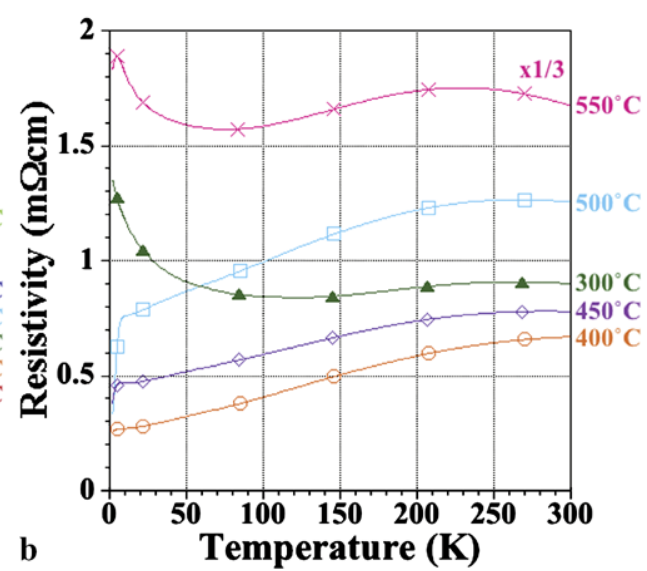

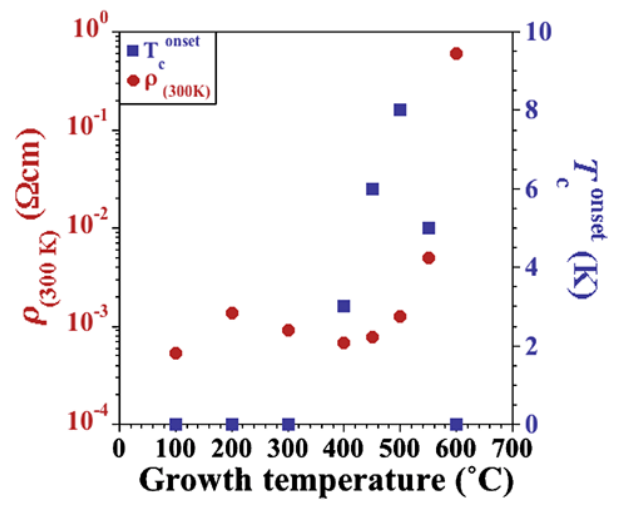

Fig. 3 The room-temperature resistivity at $300 \mathrm{~K}$ and onset temperature of the superconducting transition in resistivity ( $T_{\mathrm{c}}^{\text {onset }}$ ) of FeSe films as a function of growth temperature

in films grown at $T_{\mathrm{s}}=600^{\circ} \mathrm{C}$, though this discrepancy may be due to the difference in thickness of our thinner films $(40 \mathrm{~nm})$ in comparison to Nie's films $(200 \mathrm{~nm})$.

Figure 2 shows the temperature dependence of the resistivity. Most films show metallic behavior, and the nonsuperconducting samples show an upturn in resistivity at low temperatures. The films grown at $T_{\mathrm{S}}=600^{\circ} \mathrm{C}$ are insulating, and an onset of superconductivity is observed for films grown in the range $T_{\mathrm{s}}=400-550^{\circ} \mathrm{C}$. Figure 3 summarizes the room temperature resistivity and $T_{\mathrm{c}}$ as a function of the growth temperature. The room temperature resistivity is around $1 \mathrm{~m} \Omega \mathrm{cm}$ for films grown at $T_{\mathrm{s}}=500^{\circ} \mathrm{C}$ and increases drastically with growth temperature for $T_{\mathrm{S}}>500^{\circ} \mathrm{C}$. The $T_{\mathrm{c}}$ also depends on growth temperature and the highest $T_{\mathrm{c}}^{\text {onset }}$ was obtained for films grown at $T_{\mathrm{s}}=500^{\circ} \mathrm{C}$.

Similar to the bulk tetragonal FeSe crystals made by McQueen et al. [16] where they observe a relationship between the lattice constants and superconductivity, we also corroborate a similar trend as shown in Fig. 4, where we plot $T_{\mathrm{c}}$ versus the $c / a$ ratio for our thin films and for McQueen's bulk crystals. Although our fitted line shifts to a lower $c / a$ ratio, its slope is similar to that obtained with bulk crystals.

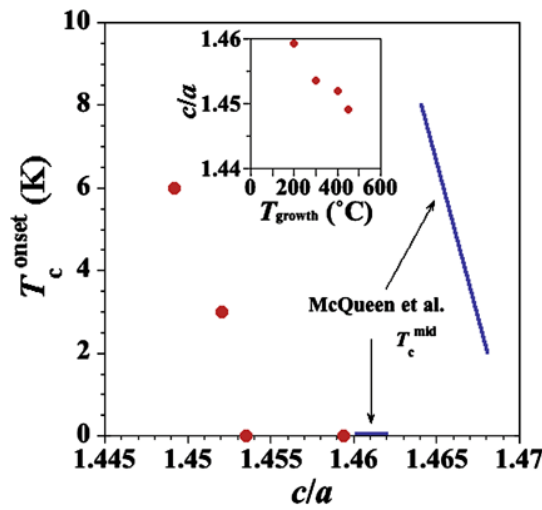

Fig. 4 The dependence of $T_{\mathrm{c}}^{\text {onset }}$ with the ratio of lattice constants $c / a$. The solid line is the result of bulk samples taken from McQueen et al. [16]. The inset shows the growth temperature dependence on the $c / a$ ratio

While the highest $T_{\mathrm{c}}^{\text {onset }}$ was obtained at a growth temperature of $T_{\mathrm{S}}=500^{\circ} \mathrm{C}$, the strongest $\left(\begin{array}{lll}0 & 0 & 1\end{array}\right)$ peak intensity from XRD measurements was obtained at $T_{\mathrm{S}}=400^{\circ} \mathrm{C}$, as shown in Fig. 5(a), where we plot both the ( $\left.\begin{array}{lll}0 & 0 & 1\end{array}\right)$ peak intensity and $T_{\mathrm{c}}^{\text {onset }}$ as a function of growth temperature. In an attempt to merge these two optimums, films were annealed in vacuum $\left(\sim 10^{-8}\right.$ torr $)$ at $500^{\circ} \mathrm{C}$ after growth at $T_{\mathrm{s}}=400^{\circ} \mathrm{C}$. Figure 5(b) shows the resistivity data for annealed films. After $60 \mathrm{~min}$ of annealing, the room-temperature resistivity increased from $0.7 \Omega \mathrm{cm}$ to $2.5 \Omega \mathrm{cm}$, both $a$ - and $c$ axis values slightly decreased from $3.791 \AA$ and $5.505 \AA$ to $3.785 \AA$ and $5.501 \AA$, respectively, but the $T_{\mathrm{c}}^{\text {onset }} \operatorname{did}$ not change ( $\sim 3 \mathrm{~K})$.

Figure 6(a) shows the valence band photoemission spectra for our FeSe films grown at various temperatures and measured in-situ by UPS. As observed in previous reports for bulk FeSe [20], the measured spectra have four distinct features (A: near $\mathrm{E}_{\mathrm{F}}, \mathrm{B}: 1.3 \mathrm{eV}, \mathrm{C}: 3.7 \mathrm{eV}$, and $\mathrm{D}: 5.7 \mathrm{eV}$ ) except for films grown at $T_{\mathrm{S}}=100^{\circ} \mathrm{C}$. Features A and B correspond to the $\mathrm{Fe}$ d-state. Feature $\mathrm{C}$ is a hybridized state of the Fe d- and Se p-states, and feature D is mainly the Se 
Fig. 5 (a) The (lo 00 1)-peak intensity and onset temperature of the superconducting transition in resistivity ( $\left.T_{\mathrm{c}}^{\text {onset }}\right)$ of FeSe films as a function of growth temperature.

(b) Temperature dependence of resistivity normalized at $20 \mathrm{~K}$ of FeSe films with post annealing at $500^{\circ} \mathrm{C}$ in vacuum
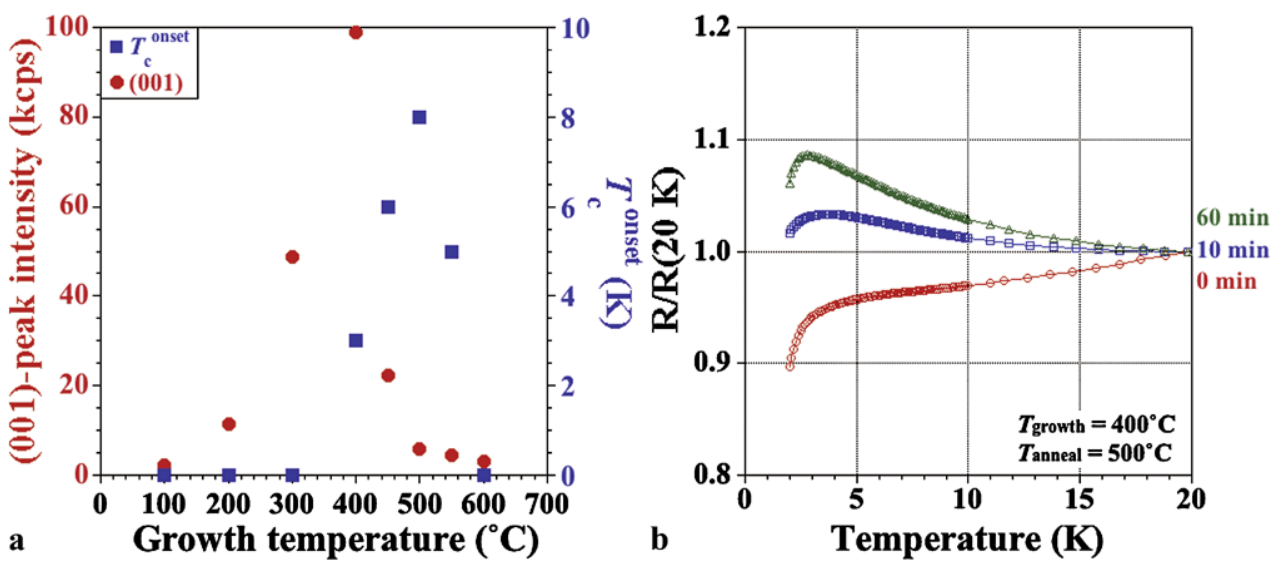

Fig. 6 The valence-band spectrum of FeSe films measured with $\mathrm{HeI}$ radiation in-situ
Fig. 7 The (a) Fe $2 p$ and (b) $\mathrm{Se}$ $3 \mathrm{~d}$ core-level XPS spectrum of FeSe films measured with $\mathrm{MgK} \alpha$ radiation in-situ
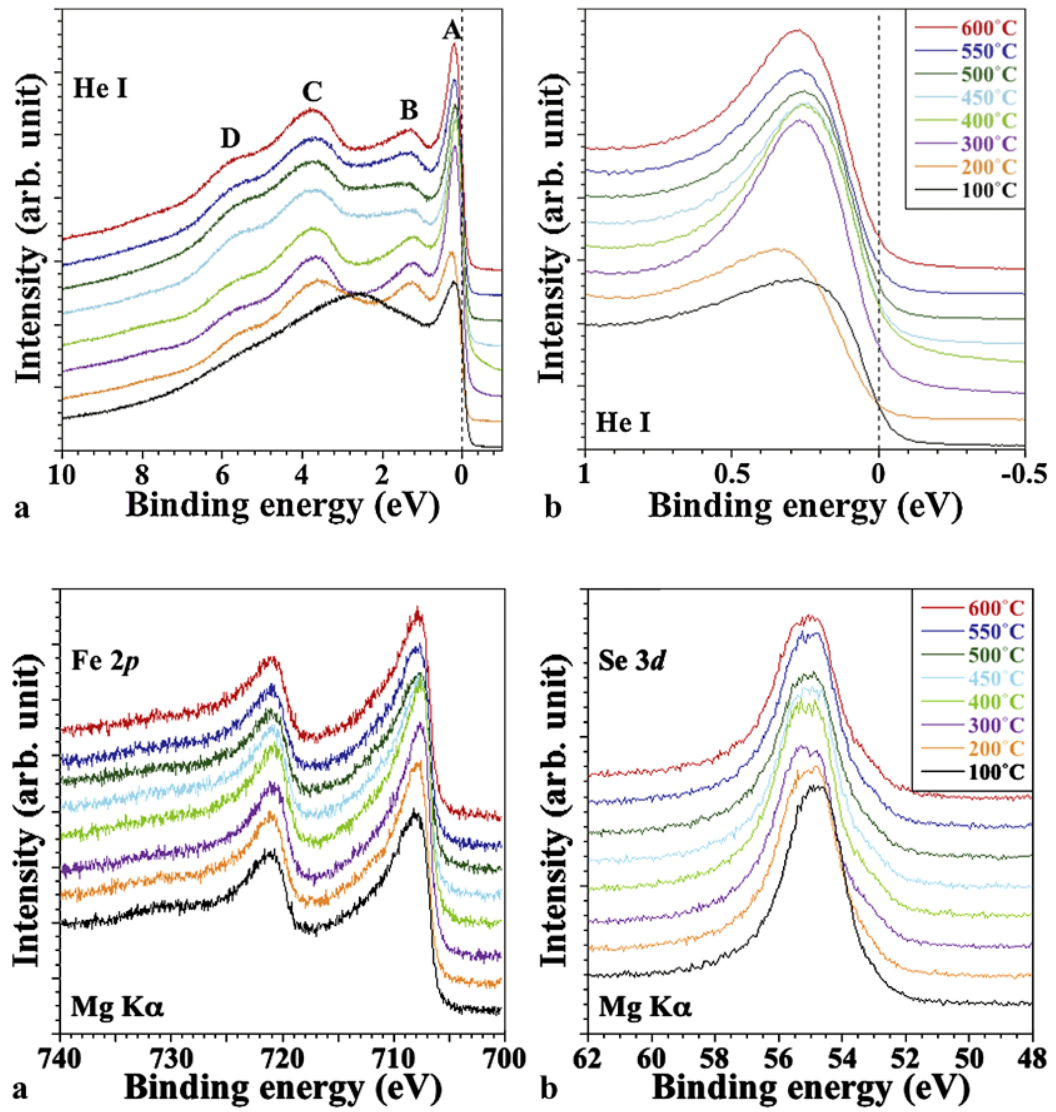

p-state. The peak positions of $\mathrm{A}, \mathrm{C}$, and D are consistent with previous reports, while the peak position of $\mathrm{B}$ is shifted to a lower binding energy by $0.7 \mathrm{eV}$. We find that the A and B peaks, which originate from $\mathrm{Fe}$, shift with growth temperature, while the $\mathrm{C}$ and $\mathrm{D}$ peaks, which originate from $\mathrm{Se}$, maintain their positions. Figure 6(b) shows the spectra near $\mathrm{E}_{F}$. A non-monotonic shift of the peak at $\sim 0.3 \mathrm{eV}$ is clearly seen.

Figure 7 shows the X-ray photoemission spectra of (a) the $\mathrm{Fe} 2 \mathrm{p}$-state and (b) the Se 3d-state for FeSe films grown at various temperatures. As shown, the Fe peaks shift with growth temperature, while the Se peak does not. While Yamasaki reported the difference of Se $3 \mathrm{~d}$ peak position between tetragonal (superconducting) FeSe and for hexagonal (non-superconducting) FeSe [21], our result shows that there is no shift of the Se 3d peak even in films grown at $T_{\mathrm{S}}=600^{\circ} \mathrm{C}$, indicating that tetragonal $\mathrm{FeSe}$ is the dominant phase in our films, as also confirmed by XRD. Figure 8 illustrates the ratio of area under the Se 3s X-ray photoemission curve to that of the $\mathrm{Fe} 3 \mathrm{p}_{3 / 2}$ curve as a function of growth temperature. The ratio between the relative areas of $\mathrm{Se}: \mathrm{Fe}$ falls within the region of 1.5-2.5. In determining this ratio, 


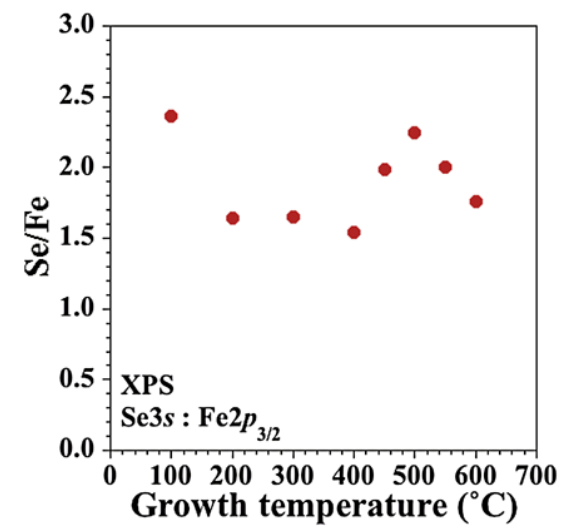

Fig. 8 The ratio of Se to $\mathrm{Fe}$ as a function of growth temperature obtained by integrating the area under the curve in XPS data for the Se $3 \mathrm{~s}$ state and $\mathrm{Fe} 2 \mathrm{p}_{3 / 2}$ state

we used the area under the main peaks of each element, but the dominance of Se in this ratio holds with other peak lines as well, including Se $3 \mathrm{~d}$ to $\mathrm{Fe} 2 \mathrm{p}$.

As shown in Figs. 1-8, the properties of our FeSe films depend strongly on growth temperature. The observed $a$ axis values indicate that our films are under tensile strain due to the lattice mismatch between LAO and FeSe. However, we stress that the large variation of the $c$-axis values cannot be understood as a consequence only of the tensile strain because the $c$-axis value varies, while the $a$-axis value remains constant. The peaks shift in XPS and UPS suggests that the doping level of $\mathrm{Fe}$ is varied with growth temperature, but even after taking into account variation of the $\mathrm{Fe} / \mathrm{Se}$ ratio, the variation of the $c$-axis value still seems large. Although we have no clear explanation for these results, it may be related to the large variation of the reported $c$-axis value for bulk samples.

We note that prior to studying the growth temperature dependence of these films, we varied the growth rate of FeSe in order to optimize its formation. Figure 9 shows the $\mathrm{X}$-ray diffraction patterns of FeSe films grown on LAO substrates at $400^{\circ} \mathrm{C}$ at different growth rates. The peaks can be indexed to $\left(\begin{array}{lll}0 & 0 \mathrm{n}\end{array}\right)$ reflections of tetragonal FeSe, indicating that the films are $c$-axis oriented. The intensities of the FeSe peaks are stronger for films grown with a growth rate of $0.11 \AA / s$ than for $0.67 \AA / s$, even at half of the thickness for the higher growth rate film, indicating that a slower growth rate yields better crystallinity. We did not explore growth rates slower than $0.11 \AA$ /s because the amount of time necessary to achieve the appropriate thickness would have caused the PLD lens inside the chamber to be significantly clouded due to the high vapor pressure of Se, which would decrease the laser intensity during the run. Additionally, the lens in the UHV chamber is not cleaned frequently as it is difficult to access, however, the lens in the HV chamber, discussed later, is cleaned frequently which is why we explored thicker

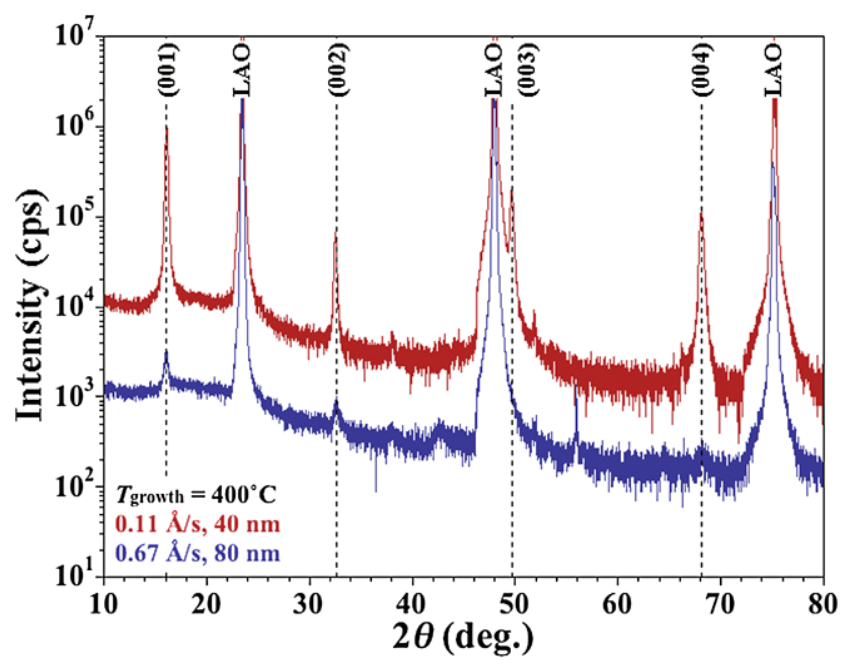

Fig. 9 X-ray diffraction patterns of films grown at $400^{\circ} \mathrm{C}$ with growth rates of $0.11 \AA / s$ and $0.67 \AA / s$

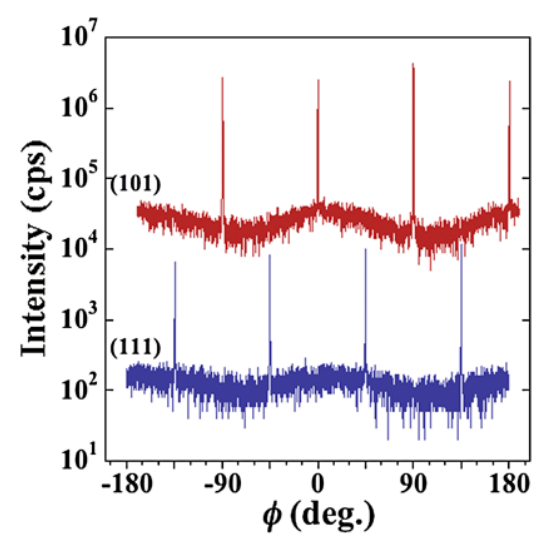

Fig. 10 The $\phi$-scan of ( $\left(\begin{array}{lll}1 & 0 & 1\end{array}\right)$ and (llll 111$)$ peaks of a typical FeSe film

films in that chamber. All our subsequent runs in the UHV chamber were performed using a $0.11 \AA$ /s growth rate.

The quality of our thin films in the UHV chamber were characterized by the $\phi$-scan XRD patterns of the (lllll) and (1 11 1) diffraction peaks in Fig. 10 and by the RHEED patterns in Fig. 11, which are typical examples of our films. The appearance of diffraction peaks $90^{\circ} \mathrm{C}$ apart in the $\phi$-scan XRD pattern indicates in-plane alignment of the FeSe film. It is interesting to note that $\mathrm{Wu}$ et al. reported the existence of a $45^{\circ} \mathrm{C}$ degree rotated domain in films grown on $\mathrm{MgO}$ substrates due to the large lattice mismatch (>10\%) [14], which underscores the importance of the choice of substrate. In-plane alignment of our FeSe films is also confirmed by RHEED (Fig. 11). Additionally, RHEED patterns along the (1 $\left.\begin{array}{lll}1 & 0\end{array}\right)$ and (1 110$)$ directions of the FeSe films grown on LAO substrates indicate a smooth surface morphology of these FeSe films.

Figure 12 shows SEM images of our films. The surface morphologies of films grown at $T_{\mathrm{s}}=400^{\circ} \mathrm{C}$ are almost the same: smooth surface with precipitates. For films grown at 

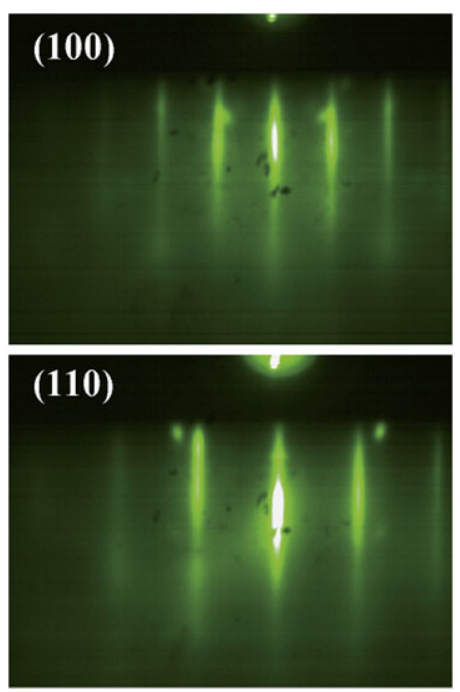

Fig. 11 RHEED patterns of an FeSe film taken along its $\left(\begin{array}{lll}1 & 1 & 0\end{array}\right)$ and (1 110$)$ direction
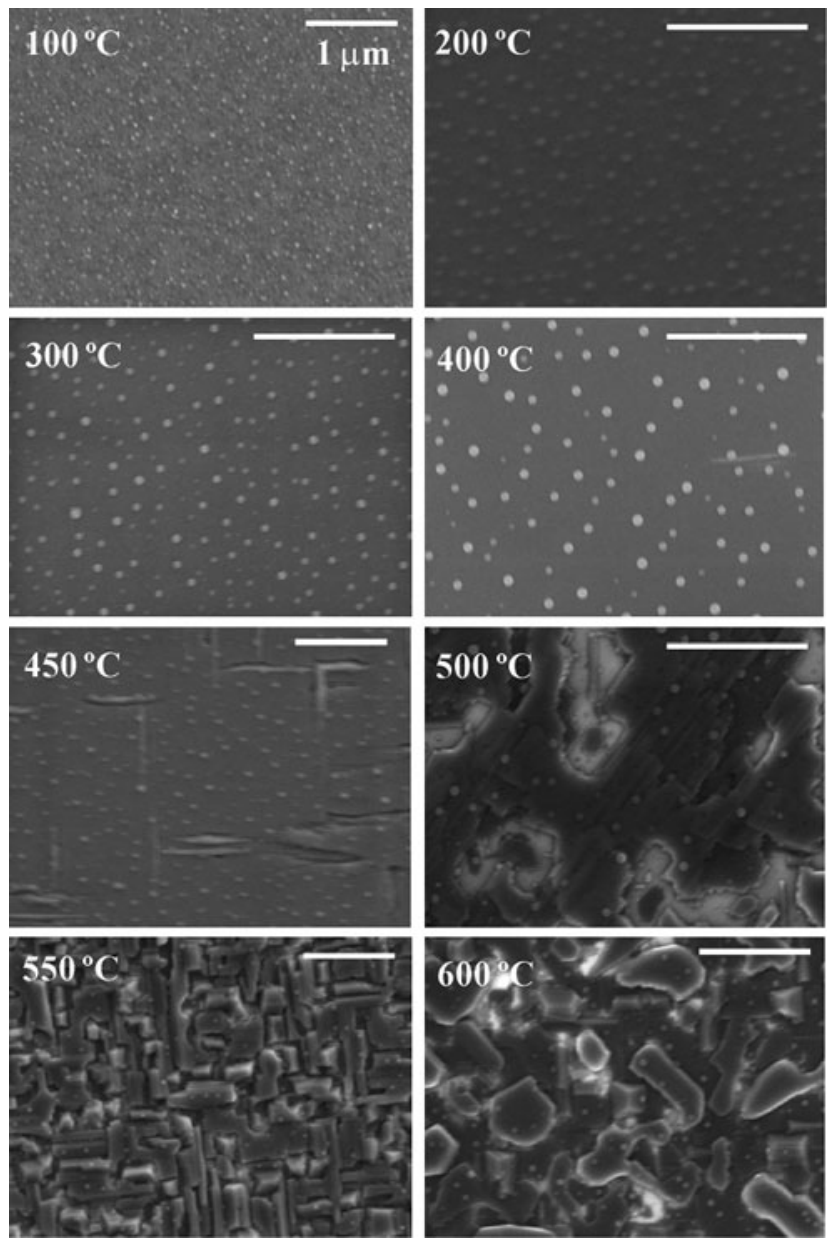

Fig. 12 SEM images of FeSe films grown at various temperatures. The length scale bar is $1 \mu \mathrm{m}$

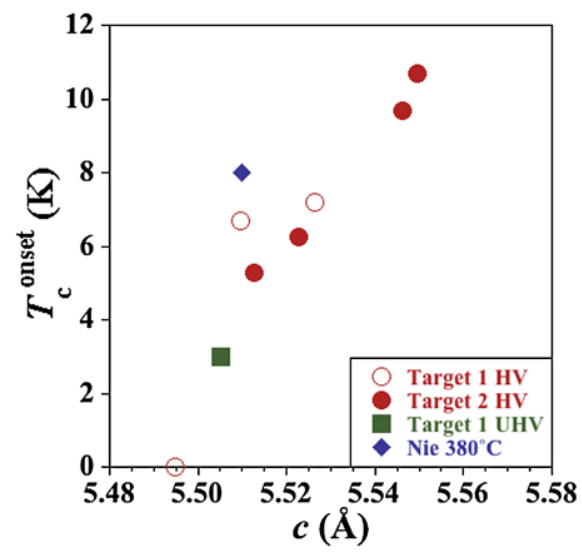

Fig. 13 Comparison of the superconducting onset temperature vs. $c$-axis lattice constant for films grown with the same target at $400^{\circ} \mathrm{C}$ in $\mathrm{UHV}$, green square, and in $\mathrm{HV}$, red open circle. Films grown at $400^{\circ} \mathrm{C}$ in HV using a second target created by using the same initial powder as the first target is also shown, red filled circle. As a comparison we also show data from Nie et al., blue diamond, where they created a $200 \mathrm{~nm}$ film grown at $380^{\circ} \mathrm{C}$ on LAO [19]

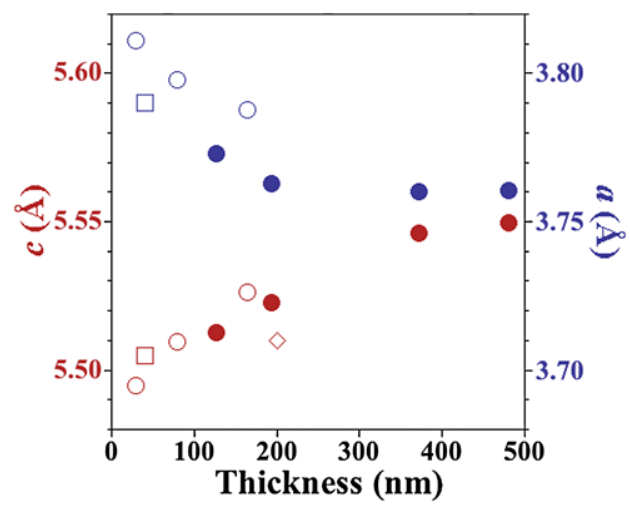

Fig. 14 Comparison of the $c$-axis and $a$-axis lattice constants with the thickness of films created. The square symbol represents a film made at $400^{\circ} \mathrm{C}$ in UHV using target 1 , the open circle represents films made at $400^{\circ} \mathrm{C}$ in $\mathrm{HV}$ also using target 1 , the filled circle represents films made at $400^{\circ} \mathrm{C}$ in $\mathrm{HV}$ using target 2 , created from the same initial powder as target 1 . We also depict data from Nie et al., open diamond, which is a $200 \mathrm{~nm}$ film grown at $380^{\circ} \mathrm{C}$ on LAO [19]

$T_{\mathrm{S}}=450^{\circ} \mathrm{C}$, some patterns are observed: layer, rectangular, and island structures for films grown at 500,550 , and $600^{\circ} \mathrm{C}$, respectively.

As we mentioned, our thickness dependence data is derived from films grown in an $\mathrm{HV}$ chamber under conditions mentioned previously. Figure 13 indicates that the $T_{\mathrm{c}}^{\text {onset }}$ increases as the thickness increases for films grown at $400^{\circ} \mathrm{C}$. Additionally, Fig. 14 shows that as the thickness increases, the strain on the film is relaxed and seems to saturate around an $a$-axis lattice constant of $3.76 \AA$. The open circles in the data indicate that the film was deposited with the same target as used in the UHV system at a laser density of $1.67 \mathrm{~J} / \mathrm{cm}^{2}$, while the filled circles indicate that a second target was used 
Fig. 15 Resistivity vs.

temperature for films of various thicknesses grown at $400^{\circ} \mathrm{C}$ in HV. Inset shows a close-up of the larger graph
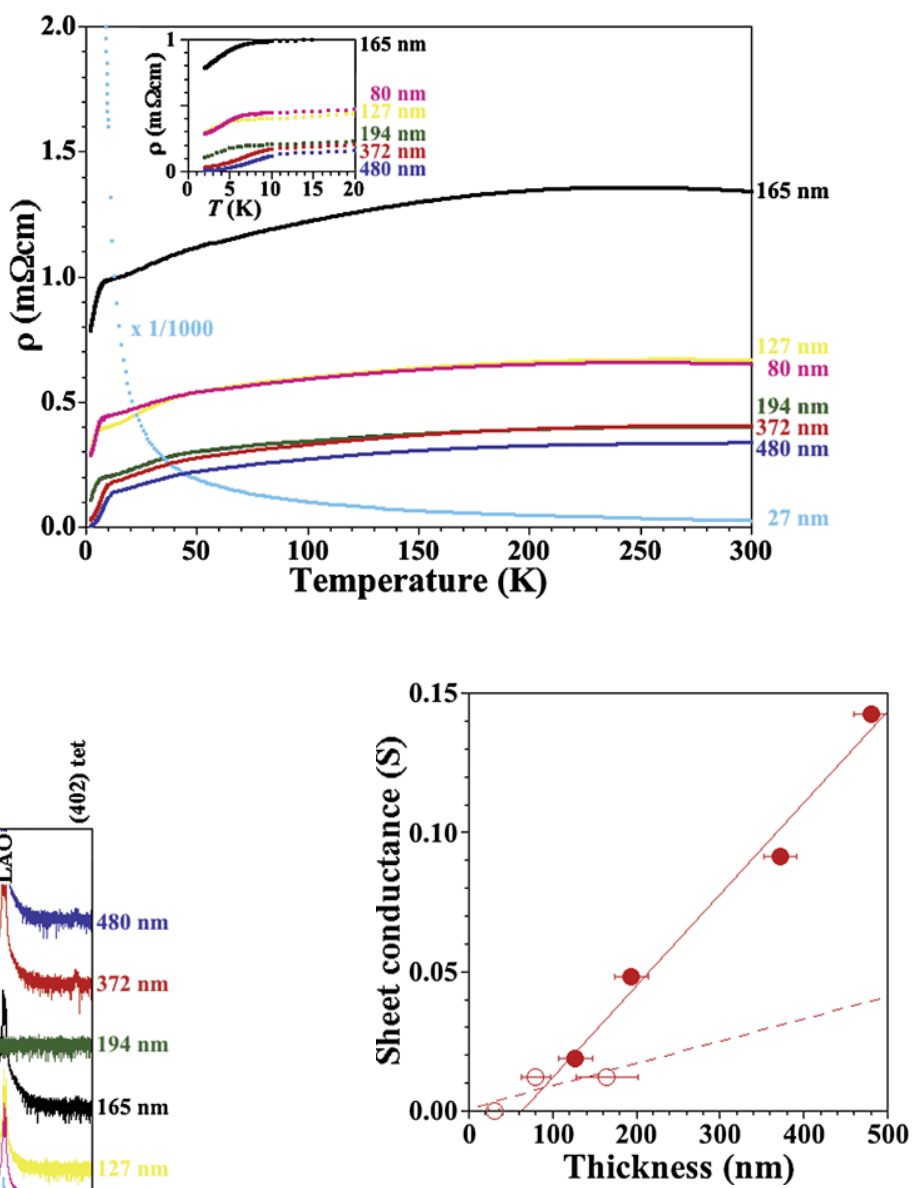

Fig. 17 Sheet conductance at room temperature for films grown at $400^{\circ} \mathrm{C}$ in $\mathrm{HV}$ at various thicknesses. The open circles represent films made with target 1 and the filled circles represent films made with target 2 , which is created from the same initial powder as target 1

Comparing the lattice constants of our thin films to that from other groups, we find that our films show the highest $T_{\mathrm{c}}^{\text {onset }}$ at a $c$-axis value of $5.5 \AA$, whereas films in previous reports show the $T_{\mathrm{c}}^{\text {onset }}$ at a $c$-axis value of $5.52 \AA$ $[14,19]$. While a consensus exists that thicker FeSe thinfilms yield better superconducting properties, there are two explanations about the effects of the thickness dependence on superconductivity either being due to a relaxation of tensile strain or to the prevention of a structural transition.

Evidence for the first suggestion, where tensile strain suppresses superconductivity, is given by Nie et al., where they grew FeSe films on $\mathrm{LAlO}_{3}, \mathrm{SrTiO}_{3}$, and $\mathrm{MgO}$ substrates and showed the relationship between the $c$-axis value and $T_{\mathrm{c}}^{\text {onset }}[19]$. They showed that thinner films $(50 \mathrm{~nm})$ have a shorter $c$-axis value (5.49-5.50 $\AA$ ) due to tensile strain and show no $T_{\mathrm{c}}^{\text {onset }}$ down to $5 \mathrm{~K}$, while thicker films $(200 \mathrm{~nm})$ have a bulk $c$-axis value $(\sim 5.52 \AA)$ and show $T_{\mathrm{c}}^{\text {onset }}$ around $8 \mathrm{~K}$ (their films do not show zero resistance down to $4 \mathrm{~K}$ ). The second explanation that epitaxy prevents FeSe from a structural transition at low temperature which leads to superconductivity is advocated by some thin film groups. But similar to the tetragonal phase [22]. 
this concept of a lack of a structural transition aiding superconductivity was initially supported by bulk crystals and pressure studies on them. In bulk crystals, McQueen et. al. reported that superconducting FeSe undergoes a structural transition from tetragonal to orthorhombic at $90 \mathrm{~K}$ but that non-superconducting FeSe does not undergo this transition [23]. With pressure, the $T_{\mathrm{c}}$ of FeSe increased to $34 \mathrm{~K}$ $[24,25]$. In applying this pressure, both $a$ - and $c$-axis values decreased and the transition of the crystal structure from tetragonal to orthorhombic was observed at room temperature. The pressure dependence of $T_{\mathrm{c}}$ showed a jump at the structural transition [25], indicating the importance of the crystal structure. With this bulk crystal perspective in mind, in thin films, Yeh et al. observed a structural transition from tetragonal to triclinic at $105 \mathrm{~K}$ [9]. Wu et al. grew FeSe films on $\mathrm{MgO}$ substrates and showed an enhancement of the $T_{\mathrm{c}}$ onset with increasing thickness ( $2 \mathrm{~K}$ for $140 \mathrm{~nm}$ and $6 \mathrm{~K}$ for $1030 \mathrm{~nm}$ with zero resistance at $4.5 \mathrm{~K}$ ) [14]. However, they mentioned that there is no large variance of the lattice constants $(a \sim 3.787 \AA$ and $c \sim 5.528 \AA$ ) with thicker films (140$1030 \mathrm{~nm})$. Hence, they explained that the strong thickness dependence of $T_{\mathrm{c}}$ is not from the lattice mismatch strain but from the prevention of a structural transition. These results indicate that tensile strain might decrease $T_{\mathrm{c}}$ but there is another parameter in determining $T_{\mathrm{c}}$, namely the suppression of a structural transition.

\section{Summary}

We have studied growth temperature dependence and thickness dependence on properties of FeSe thin films. The $a$ and $c$-axis values were strongly affected by growth temperature and thickness. The UPS and XPS measurements indicate that the doping level of $\mathrm{Fe}$ depends on growth temperature. Therefore, variation of the $a$ - and $c$-axis values with growth temperature is due to both lattice mismatch strain and variation of the $\mathrm{Fe} / \mathrm{Se}$ ratio. The superconducting transition temperature was also affected by growth temperature. Our films showed a similar $c / a$ ratio dependence on $T_{\mathrm{c}}$ as observed in bulk crystals. This result indicates that a balance between the $a$ - and $c$-axis values is important to obtain $T_{\mathrm{c}}$.

Acknowledgements This work is supported by the Department of Energy, Office of Basic Energy Science, Division of Material Sciences and Engineering, under contract DE-AC02-76SF00515.

Open Access This article is distributed under the terms of the Creative Commons Attribution Noncommercial License which permits any noncommercial use, distribution, and reproduction in any medium, provided the original author(s) and source are credited.

\section{References}

1. Y. Kamihara, T. Watanabe, M. Hirano, H. Hosono, J. Am. Chem. Soc. 130, 3296 (2008)

2. H. Hosono, J. Phys. Soc. Jpn. 77, 1 (2008)

3. K. Ishida, Y. Nakai, H. Hosono, J. Phys. Soc. Jpn. 78, 062001 (2009)

4. Z.A. Ren, W. Lu, J. Yang, W. Yi, X.L. Shen, Z.C. Li, G.C. Che, X.L. Dong, L.L. Sun, F. Zhou, Z.X. Zhao, Chin. Phys. Lett. 25, $2215(2008)$

5. C. Wang, L. Li, S. Chi, Z. Zhu, Z. Ren, Y. Li, Y. Wang, Z. Lin, Y. Luo, S. Jiang, X. Xu, G. Cao, Z. Xu, Eur. Phys. Lett. 83, 67006 (2008)

6. G. Wu, Y.L. Xie, H. Chen, M. Zhong, R.H. Liu, B.C. Shi, Q.J. Li, X.F. Wang, T. Wu, Y.J. Yan, J.J. Ying, X.H. Chen, J. Phys. Condens. Matter 21, 142203 (2009)

7. M. Rotter, M. Tegel, D. Johrendt, Phys. Rev. Lett. 101, 107006 (2008)

8. J.H. Tapp, Z. Tang, B. Lv, K. Sasmal, B. Lorenz, P.C.W. Chu, A.M. Guloy, Phys. Rev. B 78, 060505(R) (2008)

9. K.W. Yeh, H.C. Hsu, T.W. Huang, P.M. Wu, Y.L. Huang, T.K. Chen, J.Y. Luo, M.K. Wu, J. Phys. Soc. Jpn. 77, 19 (2008)

10. H. Hiramatsu, T. Katase, T. Kamiya, M. Hirano, H. Hosono, Appl. Phys. Lett. 93, 162504 (2008)

11. E. Backen, S. Haindl, T. Niemeier, R. Hühne, T. Freudenberg, J. Werner, G. Behr, L. Schultz, B. Holzapfel, Supercond. Sci. Technol. 21, 122001 (2008)

12. H. Hiramatsu, T. Kamiya, M. Hirano, H. Hosono, Physica C 469, 657 (2009)

13. H. Hiramatsu, T. Katase, T. Kamiya, M. Hirano, H. Hosono, Appl. Phys. Exp. 1, 101702 (2008)

14. M.K. Wu, F.C. Hsu, K.W. Yeh, T.W. Huang, J.Y. Luo, M.J. Wang, H.H. Chang, T.K. Chen, S.M. Rao, B.H. Mok, C.L. Chen, Y.L. Huang, C.T. Ke, P.M. Wu, A.M. Chang, C.T. Wu, T.P. Perng, Physica C 469, 340 (2009)

15. S.H. Risbud, J.R. Groza, M.J. Kim, Philos. Mag. B 69, 525 (1994)

16. T.M. McQueen, Q. Huang, V. Ksenofontov, C. Felser, Q. Xu, H. Zandbergen, Y.S. Hor, J. Allred, A.J. Williams, D. Qu, J. Checkelsky, N.P. Ong, R.J. Cava, Phys. Rev. B 79, 014522 (2009)

17. D. Phelan, J.N. Millican, E.L. Thomas, J.B. Leão, Y. Qiu, R. Paul, Phys. Rev. B 79, 014519 (2009)

18. U. Patel, J. Hua, S.H. Yu, S. Avci, Z.L. Xiao, H. Claus, J. Schlueter, V.V. Vlasko-Vlasov, U. Welp, W.K. Kwok, Appl. Phys. Lett. 94, 082508 (2009)

19. Y.F. Nie, E. Brahimi, J.I. Budnick, W.A. Hines, M. Jain, B.O. Wells, Appl. Phys. Lett. 94, 242505 (2009)

20. R. Yoshida, T. Wakita, H. Okazaki, Y. Mizuguchi, S. Tsuda, Y. Takano, H. Takeya, K. Hirata, T. Muro, M. Okawa, K. Ishizuka, S. Shin, H. Harima, M. Hirai, Y. Muraoka, T. Yokoya, J. Phys. Soc. Jpn. 78, 034708 (2009)

21. A. Yamasaki, S. Imada, K. Takase, T. Muro, Y. Kato, H. Kobori, A. Sugimura, N. Umeyama, H. Sato, Y. Hara, N. Miyakawa, S.I. Ikeda, arXiv:0902.3314v3 (2009)

22. M.M. Shivastava, O.N. Srivastava, Thin Solid Films 29, 275 (1975)

23. T.M. McQueen, A.J. Williams, P.W. Stephens, J. Tao, Y. Zhu, V. Ksenofontov, F. Casper, C. Felser, R.J. Cava, arXiv:0905.1065 (2009)

24. Y. Mizuguchi, F. Tomioka, S. Tsuda, T. Yamaguchi, Y. Takano, Appl. Phys. Lett. 93, 152505 (2008)

25. G. Garbarino, A. Sow, P. Lejay, A. Sulpice, P. Toulemonde, M. Mezouar, M. Núnez-Regueiro, Eur. Phys. Lett. 86, 27001 (2009) 